\title{
Coronary artery fistula and lung adenocarcinoma: a case report and literature review
}

\author{
Mingrui Shao ${ }^{1}$, Tao Han ${ }^{2}$, Xitao Chen ${ }^{1}$, Ziyi Wang ${ }^{1}$, Xuedong Jiang ${ }^{3}$, Wenya Li ${ }^{1}$ \\ ${ }^{1}$ Department of Thoracic Surgery, the First Affiliated Hospital of China Medical University, Shenyang, China; ${ }^{2}$ Department of Oncology, the First \\ Affiliated Hospital of China Medical University, Shenyang, China; ${ }^{3}$ Department of Thoracic Surgery, Liaoning Provincial Corps Hospital, Chinese \\ People's Armed Police Forces, Shenyang, China \\ Correspondence to: Prof. Wenya Li. Department of Thoracic Surgery, the First Affiliated Hospital of China Medical University, No. 155 North \\ Nanjing Street, Heping District, Shenyang, China. Email: saint5288@hotmail.com.
}

\begin{abstract}
Coronary artery fistula (CAF) is a rare condition, whilst lung cancer is one of the most common malignant tumors worldwide. We came cross an interesting case with both diseases. To the best of our knowledge, this is the first case report pertaining to a patient with a coexisting CAF and lung adenocarcinoma. The patient was a 67 -year-old woman who was admitted to our hospital for evaluation of persistent cough. Through the examination she was diagnosed coronary artery fistula and lung adenocarcinoma. Both diseases were successfully treated in a single operation (artery ligation and pulmonary lobectomy). The post-operative period was uneventful. At 3-month follow-up, there were no signs of blood shunting or cancer recurrence. There is no standard guidelines to treat both diseases. We want to seek out a solution to the problem. In this patient, we successfully performed artery ligation and pulmonary lobectomy in a single operation without any complications. We believe the treatment of patients with CAFs should be individualized. But, there is still a lot of shortcomings in our research. First of all, we have no enough cases to support our approach. What's more, the long-term effects of the operation are not certain. Last but not least, we have no proof in genetics with both diseases.
\end{abstract}

Keywords: Coronary artery fistula (CAF); lung adenocarcinoma; combined surgery; case report

Submitted Sep 01, 2020. Accepted for publication Jan 17, 2021.

doi: 10.21037/apm-20-2015

View this article at: http://dx.doi.org/10.21037/apm-20-2015

\section{Introduction}

Coronary artery fistula (CAF) is a rare condition that refers to an anomalous connection between one or more coronary arteries and a cardiac chamber or great vessel (1). The majority of CAFs arise from the right coronary artery (RCA), while those arising from the left anterior descending artery (LAD) and the left circumflex artery (LCx) are less common (2). Surgery is a traditional form of treatment. Transcatheter approaches may be more beneficial than surgical approaches for eligible coronary artery fistula cases. CAF can be categorized as either congenital or acquired anomalies. Congenital coronary artery fistulas are more common but only account for $0.4 \%$ of congenital cardiac abnormalities. Acquired coronary artery fistulas develop from injurious events, iatrogenic or traumatic in nature. Over the past two decades, lung adenocarcinoma has been consistently ranked as the most common type of lung cancer. So far, surgery is still the main treatment for lung adenocarcinoma. Other traditional treatments, such as chemotherapy and radiotherapy, are still effective. In recent years, new therapeutic methods such as targeted therapy and immunotherapy have emerged, and they have gained more and more effects and recognition. Cases with concomitant CAF and lung cancer have not been reported to date. Herein we report a patient with coexisting LAD-main pulmonary artery (PA) fistula and lung adenocarcinoma. Both conditions were successfully treated in a single surgical procedure.

We present the following article in accordance with 


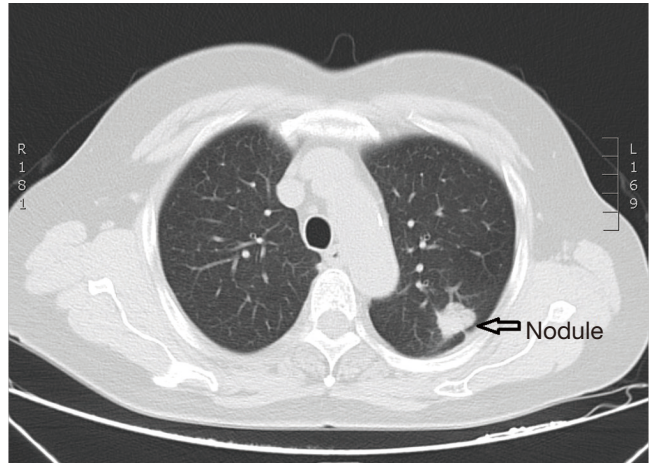

Figure 1 Computed tomography $(\mathrm{CT})$ radiograph showing a tumor measuring $2 \mathrm{~cm} \times 1.8 \mathrm{~cm}$ in the upper lobe of left lung.

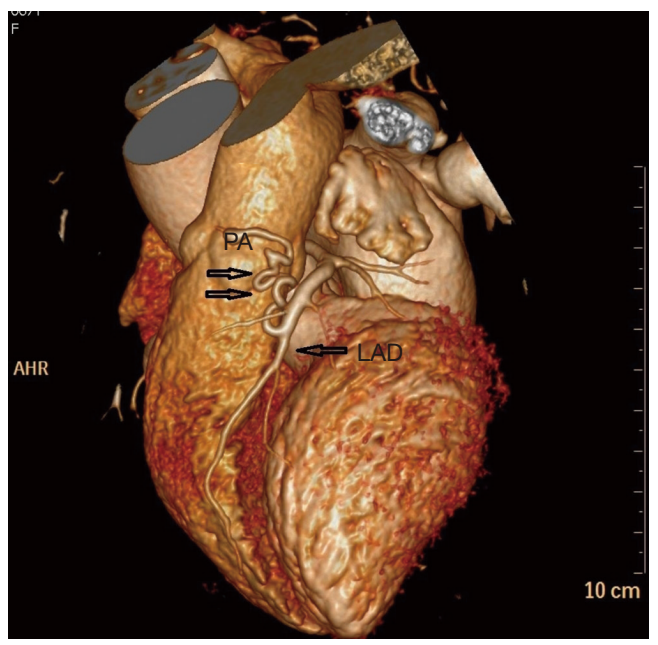

Figure 2 Three-dimensional, volume-rendered multidetector-row computed tomography (MDCT) images showing abnormal vessels located anterior to the main pulmonary artery. PA, pulmonary artery; LAD, left anterior descending artery.

the CARE reporting checklist (available at http://dx.doi. org/10.21037/apm-20-2015).

\section{Case presentation}

A 67-year-old woman was admitted to our hospital for evaluation of persistent cough. The patient was yellow and had no family history. Findings from the general physical examination were unremarkable. Laboratory investigations, including liver function tests and serum levels of carcinoembryonic antigen, carbohydrate antigen CA-199, and CA-125 were normal. Chest contrast-enhanced multidetector computed tomography (MDCT) showed a nodule

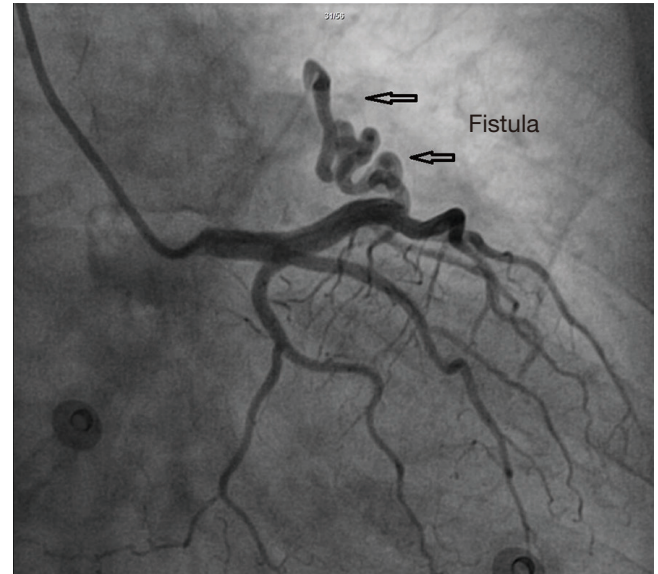

Figure 3 Coronary angiogram showing the fistula and the shunt.

(largest diameter: $2 \mathrm{~cm}$ ) in the upper lobe of the left lung (Figure 1). The tumor had a lobulated shape with spiculated margins and had caused pleural traction. The mass was suspected to be a lung carcinoma. During the pre-operative evaluation, a fistula with active flow between the LAD and the PA was detected by trans-thoracic echocardiography. The fistula was confirmed by coronary artery multi-slice spiral CT (Figure 2) and coronary angiography (Figure 3). Therefore, a diagnosis of lung neoplasm and CAF was confirmed. Lung adenocarcinoma in early stage should be treated surgically. Surgical ligation or percutaneous transcatheter occlusion are possible treatment options to coronary artery fistula. In this case, we took into account the patient's economic factors and to avoid the damage caused by the second operation. After obtaining the consent of the patient, we adopted a combined operation. We planned for surgical treatment of both conditions in a single procedure via sternotomy.

Intraoperatively, circuitous vessels originating from the mid-portion of the LAD to the PA were observed. A thrill was auscultated over the fistula vessel. We first ligated the abnormal blood vessels on the aortic and pulmonary sides. Subsequently, we opened the mediastinal pleura to visualize the left upper lobe of the lungs. The tumor was located in the periphery. A wedge resection was performed to obtain a biopsy specimen. The tumor was whitish gray in appearance. Examination of frozen sections revealed a lung adenocarcinoma. Based on the pathology report, pulmonary lobectomy and systematic mediastinal lymphadenectomy were performed. Post-operative pathologic evaluation indicated a moderately differentiated adenocarcinoma (50\% alveolar type and $40 \%$ papillary type; Figure 4 ). The pathologic TNM stage was T1bN0M0. The post-operative 


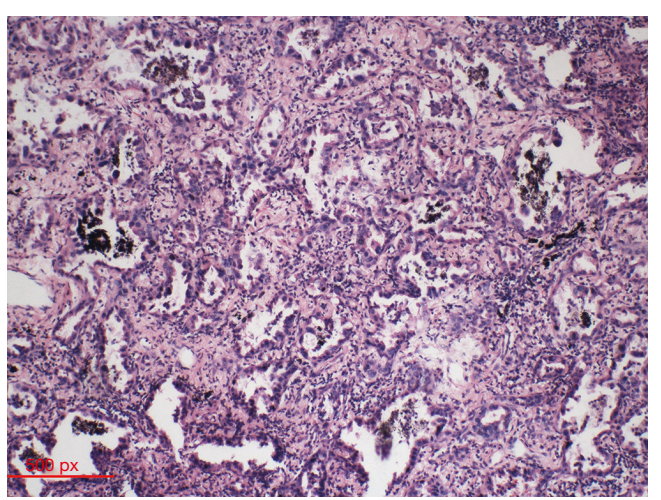

Figure 4 Microscopic examination of the surgical specimen showing moderately differentiated adenocarcinoma with $50 \%$ alveolar type and $40 \%$ papillary type (hematoxylin and eosin staining; magnification 40×).

period was uneventful and the patient was discharged on the 15 th post-operative day. No blood shunting between the coronary and pulmonary arteries or lung cancer recurrence was observed at 3-month follow-up.

All procedures performed in studies involving human participants were in accordance with the ethical standards of the institutional and/or national research committee(s) and with the Helsinki Declaration (as revised in 2013). Written informed consent was obtained from the patient.

\section{Discussion and conclusions}

CAFs were first reported by the German anatomist Krause in 1865 (3). An estimated $0.002 \%$ of the general population are affected by CAFs. Most CAFs are congenital anomalies and are typically diagnosed incidentally during coronary angiography (incidence: $0.18 \%$ ) (2). CAFs originate from the right and left coronary arteries in approximately $52-60 \%$ and $30 \%$ of cases, respectively. CAFs originating from the $\mathrm{LCx}$ account for $18 \%$ of cases (4-6). The flow endpoint of the fistula is in the right ventricle in $40 \%$ of cases, right atrium in $25 \%$ of cases, PA in $15-20 \%$ cases, coronary sinus in $7-10 \%$ of cases, and the superior vena cava in $1-2 \%$ cases (7).

Most CAFs are congenital, however, CAFs may also occur as a complication of chest trauma, infection, coronary angioplasty, or cardiac operations $(8,9)$. Clinical symptoms arising from CAFs are unusual. Most patients are asymptomatic for long periods of time (10). Some congenital CAFs can be identified in childhood based on murmurs and/or clinical symptoms (11). Symptoms are more likely to manifest in the elderly, and are largely attributable to the blood flow through the shunt and the structures involved (12-15). CAFs may sometimes cause acute myocardial infarction, which may necessitate coronary artery bypass surgery $(7,16)$. Our patient had no apparent symptoms because there was a minimal shunt from the distal coronary blood flow.

Coronary angiography and MDCT are reliable techniques used to identify and delineate the course of anomalous coronary arteries (17). Other examinations, such as magnetic resonance imaging (MRI), transthoracic echocardiography, and Doppler echocardiography are also helpful (18-21). In the present case, CAF was detected during trans-thoracic echocardiography as part of the pre-operative evaluation for lung neoplasm, and was subsequently confirmed by coronary angiography.

There are no standard guidelines for the treatment of CAFs (22). The indications for fistula closure are mainly based on clinical symptoms, such as heart failure, myocardial ischemia, aneurysmal dilatation, or high-flow shunting $(23,24)$. In severe cases, spontaneous rupture of coronary artery-to-pulmonary artery fistulas may cause hemopericardium and cardiac tamponade (25). Current treatment options include observation, transcatheter embolization, and surgical ligation. Transcatheter approaches are preferred over the open surgical approach owing to the lower cost, lower morbidity, shorter recovery time, and better cosmetic results. Transcatheter techniques also have some shortcomings, such as distal embolization, which may cause ischemic electrocardiographic changes or myocardial infarction (26). Surgical closure is preferred for CAFs with the following characteristics: (I) locations inaccessible for percutaneous intervention; (II) multiple fistulae; (III) extreme native vessel tortuosity; (IV) aneurysmal dilation of the fistula or proximal coronary artery; and $(\mathrm{V})$ presence of normal coronary branches near the fistula drainage sites (27). Biorck and Crafoord (28) were the first to report open surgery in a patient with a coronary artery-to-pulmonary artery fistula in 1947 . Our case was unusual because the patient had two different medical problems (coronary artery-to-pulmonary artery fistula and lung adenocarcinoma). The clinical pre-operative TNM staging was T1bN0M0, for which surgical resection is the standard treatment. We decided to treat both conditions in a single operation. No complications happened after the surgery. The pathologic TNM stage was T1bN0M0, so we didn't do chemotherapy. The patient recovered well and was discharged 15 days after surgery, thus avoiding a second surgical procedure. 
In summary, we have reported the first case of concomitant $\mathrm{CAF}$ and lung cancer. Our successful experience suggests that the treatment of patients with CAFs should be individualized.

\section{Acknowledgments}

Funding: This study was supported by Natural Science Foundation of Liaoning Province (grant No. 2015020561), the Fund for Scientific Research of The First Hospital of China Medical University (grant No. fsfh 1514) and Wu Jieping Medical Foundation (grant No. 320.6750.18293).

\section{Footnote}

Reporting Checklist: The authors have completed the CARE reporting checklist. Available at http://dx.doi.org/10.21037/ apm-20-2015

Conflicts of Interest: All authors have completed the ICMJE uniform disclosure form (available at http://dx.doi. org/10.21037/apm-20-2015). The authors have no conflicts of interest to declare.

Ethical Statement: The authors are accountable for all aspects of the work in ensuring that questions related to the accuracy or integrity of any part of the work are appropriately investigated and resolved. All procedures performed in studies involving human participants were in accordance with the ethical standards of the institutional and/or national research committee(s) and with the Helsinki Declaration (as revised in 2013). Written informed consent was obtained from the patient.

Open Access Statement: This is an Open Access article distributed in accordance with the Creative Commons Attribution-NonCommercial-NoDerivs 4.0 International License (CC BY-NC-ND 4.0), which permits the noncommercial replication and distribution of the article with the strict proviso that no changes or edits are made and the original work is properly cited (including links to both the formal publication through the relevant DOI and the license). See: https://creativecommons.org/licenses/by-nc-nd/4.0/.

\section{References}

1. Vavuranakis M, Bush CA, Boudoulus H. Coronary artery fistula in adults: incidence, angiographic characteristics, natural history. Cathet Cardiovasc Diagn 1995;35:116-20.
2. Bruhin R, Stock UA, Breuer M, et al. Successful in situ repair of a symptomatic left main coronary artery aneurysm by a saphenous vein graft. Interact Cardiovasc Thorac Surg 2004;3:434-6.

3. Krause W. Ueber den Ursprung einer accesorischen A. Coronaria cordis aus der A. Pulmonalis. Z Ratt Med $1865 ; 24: 255$.

4. Tiryakioglu SK, Gocer H, Tiryakioglu O, et al. Multiple coronary-cameral fistulae. Tex Heart Inst J 2010;37:378-9.

5. McNamara JJ, Gross RE. Congenital coronary artery fistula. Surgery 1969;65:59-69.

6. Qureshi SA. Coronary arterial fistulas. Orphanet J Rare Dis 2006; 1:51.

7. Talay S, Saracoglu T, Kabalar ME, et al. Urgent coronary artery bypass graft operation of a patient with acute anterior myocardial infarction, congenital right coronary artery to main pulmonary artery fistula and left hilar hamartoma of the lung. Eur Surg 2011;5:315-7.

8. Gupta A, Saxena S. Acquired (post-angioplasty) coronary ventricular fistula draining into left ventricle aneurysm. J Invasive Cardiol 2012;24:E99-100.

9. Almendro-Delia M, Ortiz-Cortes C, CarrascosaRosillo C. Coronary arteriovenous fistula secondary to iatrogenic coronary perforation. Rev Esp Cardiol (Eng Ed) 2014;67:490.

10. Luo L, Kebede S, Wu S, et al. Coronary artery fistulae. Am J Med Sci 2006;332:79-84.

11. Hsieh KS, Huang TC, Lee CL. Coronary artery fistulas in neonates, infants, and children: clinical findings and outcome. Pediatr Cardiol 2002;23:415-9.

12. Mavroudis C, Backer CL, Rocchini AP, et al. Coronary artery fistulas in infants and children: a surgical review and discussion of coil embolization. Ann Thorac Surg 1997;63:1235-42.

13. Kamiya H, Yasuda T, Nagamine H, et al. Surgical treatment of congenital coronary artery fistulas: 27 years' experience and a review of the literature. J Card Surg 2002;17:173-7.

14. Wang S, Wu Q, Hu S, et al. Surgical treatment of 52 patients with congenital coronary artery fistulas. Chin Med J 2001;114:752-5.

15. Fernandes ED, Kadivar H, Hallman GL, et al. Congenital malformations of the coronary arteries: the Texas Heart Institute experience. Ann Thorac Surg 1992;54:732-40.

16. Said SAM, de Voogt WG, Hamad MS, et al. Surgical treatment of bilateral aneurysmal coronary to pulmonary artery fistulas associated with severe atherosclerosis. Ann Thorac Surg 2007;83:291-3. 
17. Kang WC, Moon CI, Ahn TH, et al. Identifying the course of a coronary-bronchial artery fistula using contrastenhanced multi-detector row computed tomography.Int J Cardiol 2008;130:e125-8.

18. Tsubata S, Ichida F, Miyawaki T. Noninvasive diagnosis of coronary arterial fistulas using multiplanar cine magnetic resonance imaging. Cardiol Young 1998;8:250-2.

19. Giannoccaro PJ, Sochowski RA, Morton BC, et al. Complementary role of transoesophageal echocardiography to coronary angiography in the assessment of coronary artery anomalies. Br Heart J 1993;70:70-4.

20. Dawn B, Talley J, Prince C, et al. Two-dimensional and Doppler transesophageal echocardiographic delineation and flow characterization of anomalous coronary arteries in adults. J Am Soc Echocardiogr 2003;16:1274-86.

21. Woo JJ, Koh YY, Ha SI, et al. Non-invasive recognition of generalized coronary arteriosystemic fistulae by contrast echocardiography and multidetector CT. Int J Cardiovasc Imaging 2011;27:749-53.

Cite this article as: Shao M, Han T, Chen X, Wang Z, Jiang X, Li W. Coronary artery fistula and lung adenocarcinoma: a case report and literature review. Ann Palliat Med 2021;10(1):754758. doi: 10.21037/apm-20-2015
22. Hong GJ, Lin CY, Lee CY, et al. Congenital coronary artery fistulas: clinical considerations and surgical treatment. ANZ J Surg 2004;74:350-5.

23. Angelini P. Are all fistulae worth closing? Editorial comment. Tex Heart Inst J 2005;32:58-9.

24. Raju MG, Goyal SK, Punnam SR, et al. Coronary artery fistula: a case series with review of the literature. J Cardiol 2009;53:467-72.

25. Bauer HH, Allmendinger PD, Flaherty J, et al. Congenital coronary arteriovenous fistula: spontaneous rupture and cardiac tamponade. Ann Thorac Surg 1996;62:1521-3.

26. Loukas M, Germain AS, Gabriel A, et al. Coronary artery fistula: a review. Cardiovasc Pathol 2015;24:141-8.

27. Latson LA. Coronary artery fistulas: How to manage them. Catheter Cardiovasc Interv 2007;70:110-16.

28. Biorck G, Crafoord C. Arteriovenous aneurysm on the pulmonary artery simulating patent ductus arteriosus Botalli. Thorax 1947;2:65-74.

(English Language Editor: C. Betlazar-Maseh) 